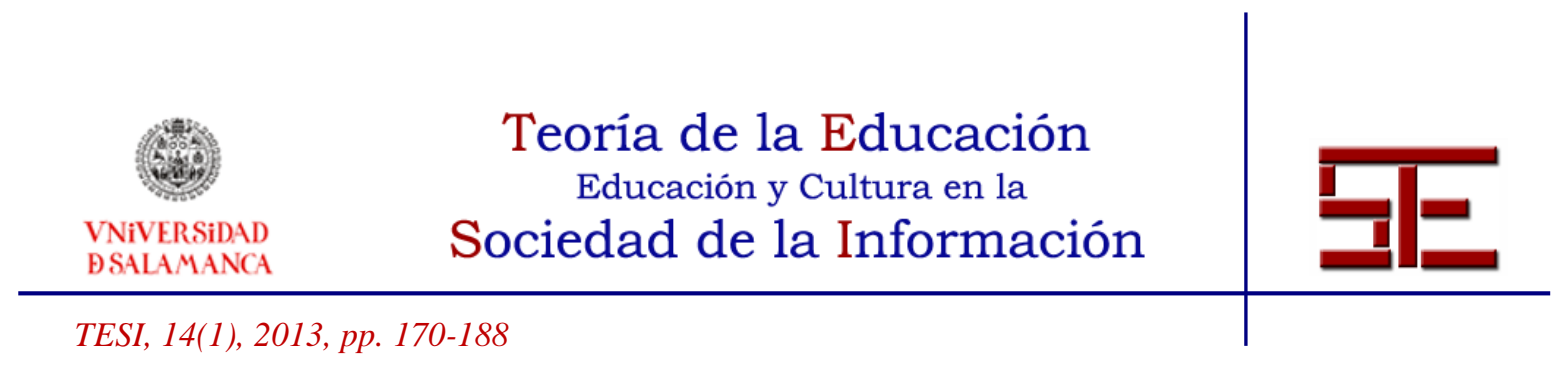

MISCELÁNEA

\title{
TÉCNICAS DE AYUDA EN EL PROCESO DE APRENDIZAJE: EL CASO DE LOS SISTEMAS ANTICOPIA*
}

Resumen: La extensión del uso de Internet ha proporcionado a los estudiantes la posibilidad de acceder a fuentes de información no disponibles en el pasado. Este hecho ha incrementado el uso inadecuado de la información obtenida. En este trabajo analizamos la experiencia de uso de una licencia del sistema antiplagio Turnitin. En total fueron recopilados 350 trabajos entregados para su análisis. Del mismo pudimos percibir un efecto aprendizaje entre entrega y entrega. Las principales conclusiones aportadas tienen un efecto positivo. Así, hemos comprobado como el alumno toma conciencia de la existencia de Turnitin, lo que ha aumentado su dedicación al trabajo escrito, al mismo tiempo que ha disminuido las sospechas sobre las entregas del resto de estudiantes, dando valor adicional al trabajo entregado, reforzando la relación con el profesor y reforzando su autoridad. La evaluación del uso de Turnitin también ha mostrado ciertos aspectos negativos: la aparición de una sensación de rechazo por la percepción de un control excesivo, la adaptación del trabajo escrito a los requisitos de Turnitin y el vacío legal que existe en la distribución de los trabajos de los estudiantes.

Palabras clave: Proceso de aprendizaje; plagio; Turnitin; ética universitaria.

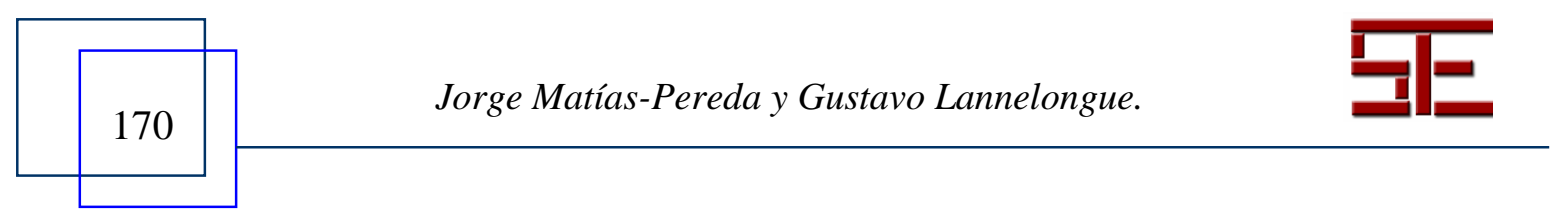




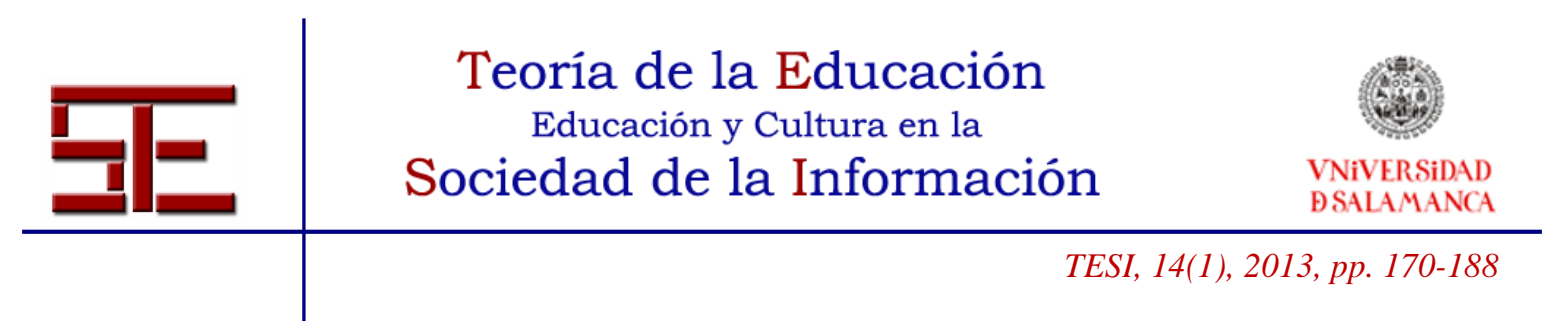

\section{SUPPORT TECHNIQUES IN THE LEARNING PROCESS: THE CASE OF ANTIPLAGIARISM SYSTEMS}

Abstract: The widespread use of the Internet has given university students access to information resources on a level never experienced in the past. The bad news is that this issue increased the inappropriate use of those resources. In this paper we discuss the experience of using the Turnitin anti-plagiarism license. In total, 358 assignments were analyzed. We partly noticed a learning effect among students between assignment deliveries. The main conclusions drawn from the use of Turnitin anti-plagiarism license are positive and we have seen how students have become aware of the existence of this tool in the evaluation process, which in turn has led to greater attention and dedication to the writing process and to the development of ideas and concepts. It has also allowed to diminish suspicions about the authenticity of the work handed by the students, valuing the real effort, improving their relationship with the teacher reinforcing the authority of the latter. The incorporation of the license can also lead to some negative aspects: The generation of a feeling of rejection by the student by the exaggerated perception of control, the adaptation of written work to Turnitin requirements and the legal vacuum surrounding the dissemination of student work.

Keywords: Learning processes; Plagiarism; Turnitin; Ethics in higher education.

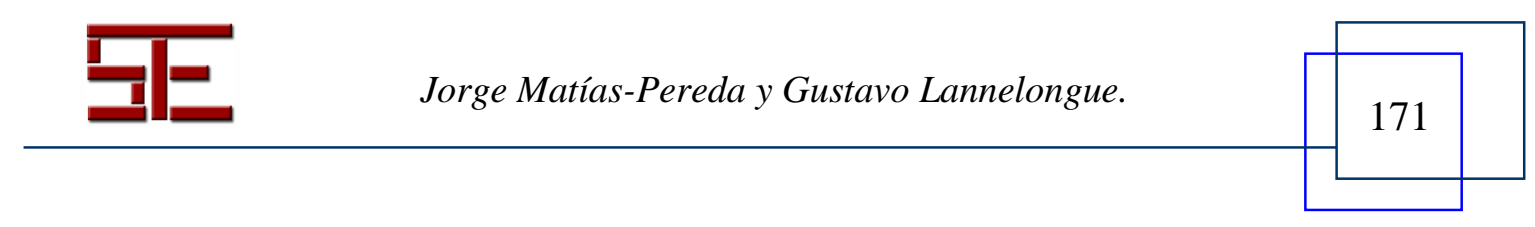




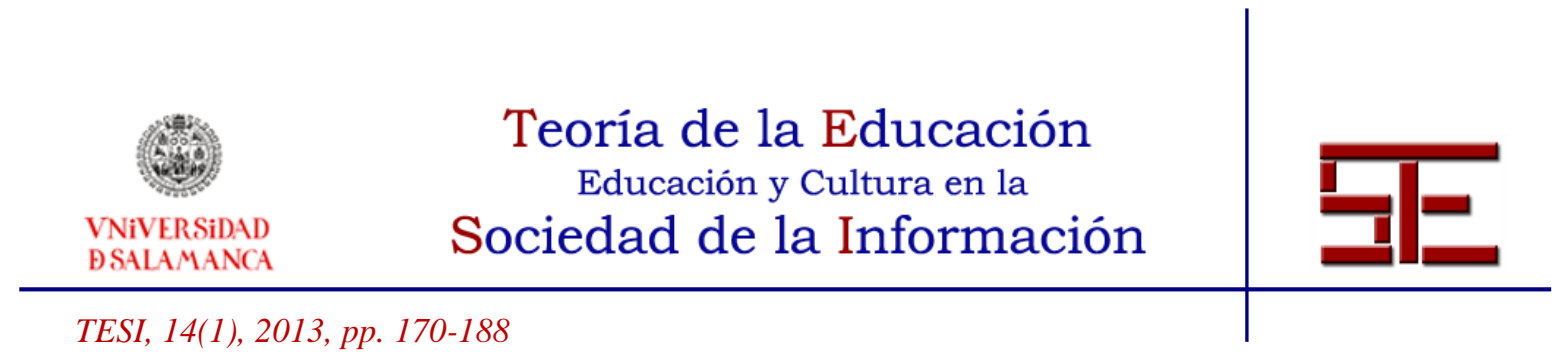

\section{TÉCNICAS DE AYUDA EN EL PROCESO DE APRENDIZAJE: EL CASO DE LOS SISTEMAS ANTICOPIA}

Fecha de recepción: 30/05/2012; fecha de aceptación: 15/01/2013; fecha de publicación: 28/02/2013

Jorge Matías-Pereda

mpereda@usal.es

Universidad de Salamanca

Gustavo Lannelongue Nieto

lannelongue@usal.es

Universidad de Salamanca

\section{1.- INTRODUCCIÓN}

La Universidad española se adentra poco a poco en una reforma de calado que pretende facilitar la movilidad, preparar a sus alumnos para la vida profesional y el aprendizaje continuo, y ofrecer el acceso a una educación superior de calidad (Declaración de Bolonia, 1999). El nuevo enfoque educativo, basado en el desarrollo de competencias, requiere de procesos de evaluación, en su esencia, muy distintos de los aplicados hasta el momento.

En este estado de cosas, el conocimiento no es más un elemento transmisible, sino que es el propio sujeto, con su propia acción, el que determina los caminos de aprendizaje a través de su experiencia vital. Desde esta perspectiva, el docente es un constructor de espacios para la experimentación y el aprendizaje activo del alumno.

Qué duda cabe de que la extensión del uso de Internet como soporte del contenido de trabajos y ensayos, y una divulgación mayor de ciertas prácticas poco adecuadas, tales como el "corta y pega" (Douglas, 2004), unido a la conexión permanente de los alumnos entre sí por medio de redes sociales, hace que el docente tenga que replantearse su función de supervisor ético en el marco universitario.

Para hacer valer el trabajo del docente y dar un verdadero sentido a su labor, es necesario el establecimiento de esa supervisión ética. De otro modo, la función docente perdería su sentido y su reconocimiento social.

Para ayudar a cumplir esa misión del docente existen diversas herramientas que están teniendo un auge considerable, sobre todo en el mundo anglosajón, es el conjunto de herramientas denominadas anticopia o antiplagio. En este capítulo relataremos la experiencia de la utilización de la licencia anticopia Turnitin, utilizada primero de forma

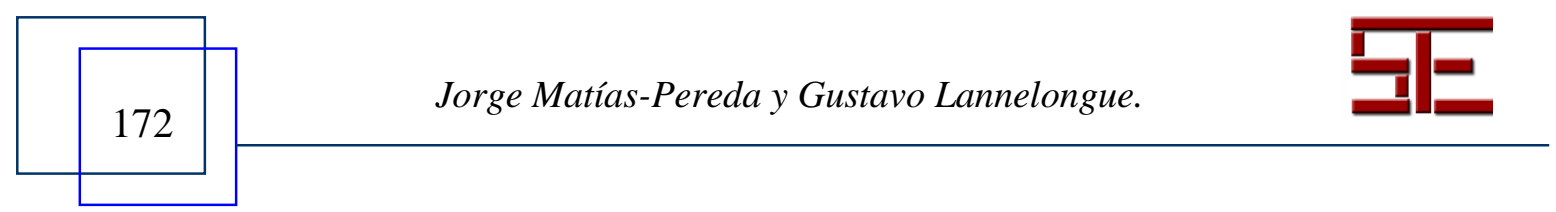




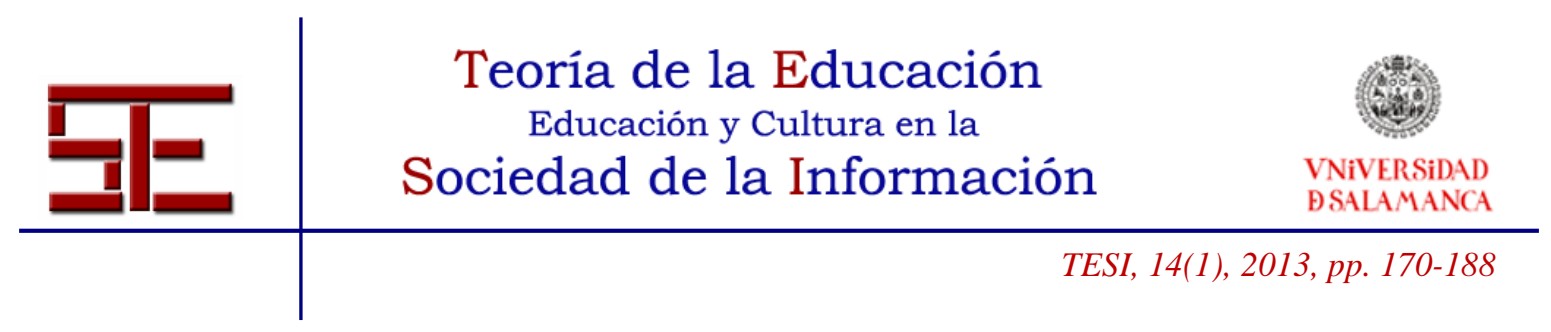

experimental y, posteriormente, de forma completa con la adquisición de una licencia para 300 alumnos en la Facultad de Economía y Empresa de la Universidad de Salamanca.

En la descripción de la presente experiencia del uso de Turnitin, comenzaremos describiendo el papel que la tecnología juega en el proceso de aprendizaje dentro del marco del Espacio Europeo de Educación Superior y, más particularmente, en las asignaturas de Organización de Empresas y de Dirección Estratégica. En el siguiente apartado se describe el sistema anticopia Turnitin y su funcionamiento. Posteriormente se hace una comparación de las herramientas libremente disponibles para el docente en la lucha contra la copia frente al sistema Turnitin aquí descrito. Pasando después a describir los resultados prácticos de la aplicación en el aula para un total de cuatro grupos de clase, tres asignaturas y 179 alumnos. A la luz de la experiencia se enumeran y describen las ventajas e inconvenientes encontrados en el uso de Turnitin, para terminar con un apartado de conclusiones que incluye una serie de recomendaciones para obtener un mejor rendimiento del sistema.

\section{2.- EL PROCESO DE APRENDIZAJE DENTRO DEL MARCO DEL EEES: LA IMPORTANCIA DE LA TECNOLOGÍA}

El EEES tiene fundamentalmente dos objetivos: uno, la homogeneización de los títulos de educación superior para fomentar la movilidad de los estudiantes, profesores y egresados; y dos, la medición del proceso de aprendizaje mediante un sistema unificado de créditos (ECTS) que tenga en cuenta el tiempo que el estudiante dedica a cada asignatura, lo cual simplifica las convalidaciones y crea la estructura educativa adecuada para desarrollar el primer objetivo. Esto implica un cambio en la estructura de las titulaciones académicas y, además, un cambio metodológico en el proceso de enseñanza en el que se reducen las clases magistrales presenciales en beneficio de seminarios y actividades tutorizadas en grupos más reducidos.

En paralelo a estos profundos cambios en nuestro sistema, las agencias de acreditación y las universidades están tratando de incorporar ítems relacionados con la calidad de la docencia en las acreditaciones profesionales y en el acceso a puestos académicos. En este contexto, la adaptación al EEES de las asignaturas de dirección estratégica supone un reto y una oportunidad para los docentes, en la que la tecnología puede jugar un papel muy importante, fundamentalmente por tres aspectos:

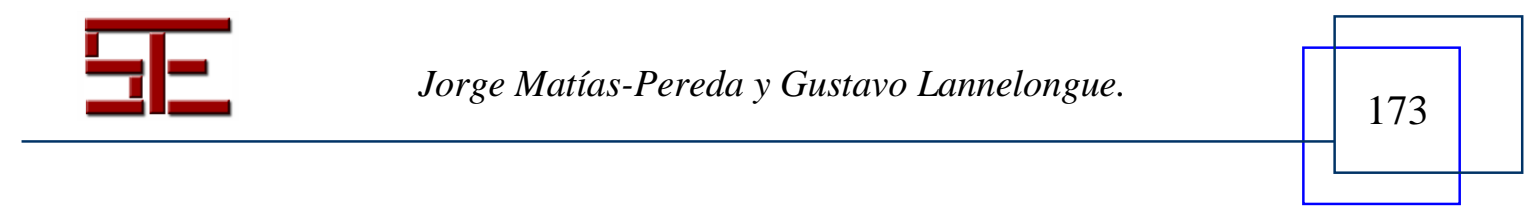




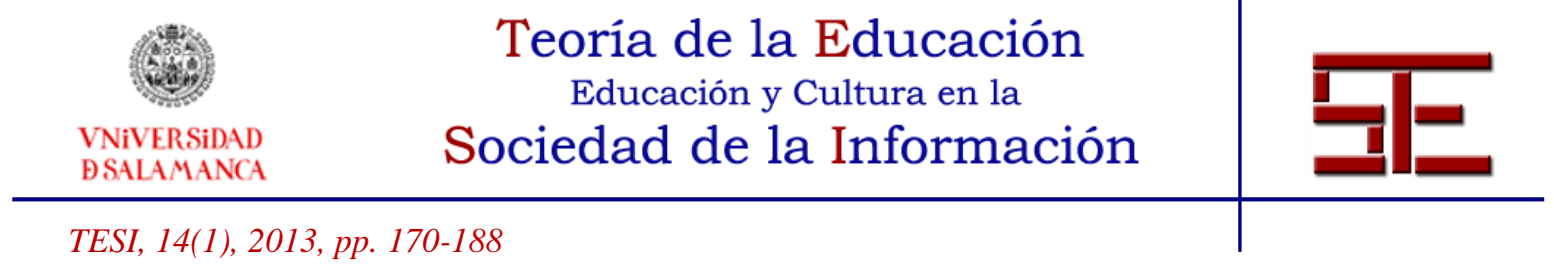

Primero, por el cambio de rol del profesor pasando de ser un transmisor de conocimiento a ser más un mediador en la construcción del conocimiento por parte de cada alumno. Esto requiere un mayor esfuerzo de supervisión del proceso de aprendizaje por parte del profesor que necesitará apoyarse en las Tecnologías de la Información y la Comunicación para facilitar esa supervisión.

Segundo, por el cambio de rol del alumno, que pasa de ser un actor pasivo a un rol activo en su proceso de aprendizaje, en el que va a tener mayor libertad para gestionar el tiempo dedicado al estudio de la asignatura.

Tercero, por los cambios metodológicos que deberán tender a aumentar las posibilidades de contacto con el profesor y entre los alumnos. El uso del aula virtual, con sus foros o chats, o el propio correo electrónico, permiten y fomentan estos contactos.

Los docentes de Dirección Estratégica nos encontramos con el problema de mantener un comportamiento ético del alumnado, que repercute directamente en la evaluación correcta y ajustada al rendimiento real, amén de estar cumpliendo el objetivo de garantizar una educación integral de aquellos que serán el soporte de la sociedad del mañana inmediato. Los nuevos alumnos son ágiles usuarios de Internet y se encuentran habituados desde la educación secundaria a utilizar la red para obtener información en la que soportar sus actividades académicas, especialmente la confección de ensayos y prácticas. Hay un conjunto de herramientas disponibles en el mercado para mitigar el problema: los sistemas anticopia. Basados en algún tipo de comparación entre un documento particular (trabajo, ensayo o práctica) y un conjunto más o menos extenso de documentos accesibles desde Internet, pretenden ofrecer una herramienta que dé solución a la problemática antes descrita.

\section{3.- EL SISTEMA ANTICOPIA TURNITIN}

Turnitin es una de las herramientas disponibles comercialmente, dentro de la categoría de sistemas anticopia, que nos permite conocer si el trabajo de un alumno presenta puntos de coincidencia con textos de autoría distinta a la del propio alumno, su localización y acceso a las fuentes sobre las que se presentan los indicios de plagio. Sin duda es un salto cualitativo importante el poder realizar, prácticamente de forma automática, una comprobación de similitud de los trabajos que el docente recibe para su calificación. Es una forma más, enfocada a mejorar la productividad y la calidad del trabajo del docente

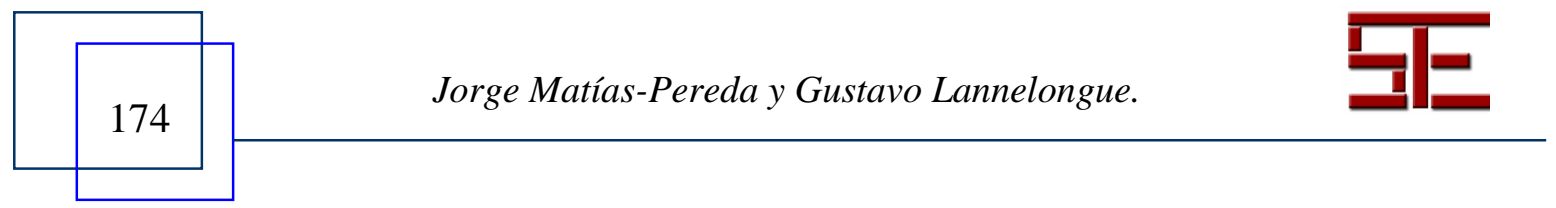




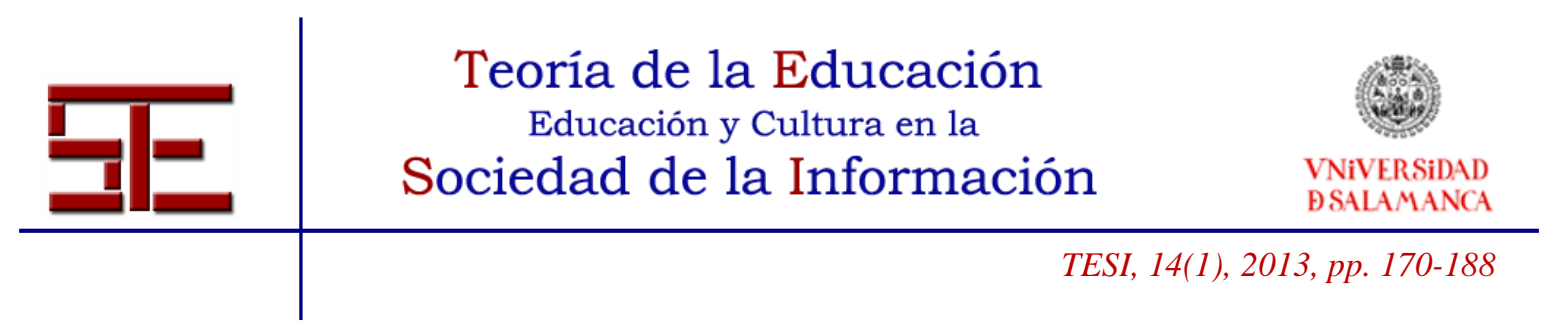

en el aseguramiento de la autenticidad del trabajo del alumno, base de su proceso de aprendizaje supervisado.

Turnitin se encuentra muy extendido en el mundo académico, ya que es utilizado por más de 800.000 docentes, 9.500 instituciones académicas en 126 países y 19 millones de alumnos (www.turnitin.com) y es uno de los sistemas de referencia en la lucha contra el plagio en estudios de educación secundaria y universitarios de grado y posgrado.

Las asignaturas en las que se ha aplicado la licencia y que son objeto del presente trabajo han sido: Dirección de la Producción, Dirección de la Información (ambas asignaturas troncales pertenecientes al tercer curso de la licenciatura en Administración y Dirección de empresas) e Información, Organización y Control (asignatura optativa perteneciente al cuarto curso en la licenciatura en Administración y Dirección de Empresas).

\section{1.- Funcionamiento del sistema}

Una vez el profesor ha generado el espacio correspondiente a una asignatura concreta en la aplicación Turnitin, se deben generar carpetas por cada uno de los trabajos que se quieran analizar con la licencia. El código que asigna Turnitin a cada alumno es único y la institución paga por cada una de estas licencias individuales en función del número de alumnos totales. Es por ello que es más ventajoso económicamente contratar una licencia a nivel de toda la universidad, al mismo tiempo que se facilitan las tareas de integración en los campus virtuales y el trabajo administrativo.

Una vez dentro de la asignatura en la plataforma Turnitin, el alumno puede, antes de enviar el trabajo, ver las notas e indicaciones que el profesor ha querido destacar, como por ejemplo el formato admitido, la extensión del trabajo, la fecha límite, etc. Cuando el alumno sube el trabajo, Turnitin hace una copia exacta del mismo para comprobar si el formato del trabajo puede ser comparado con el sistema de verificación. El alumno recibe entonces un número de registro, la copia de su trabajo y el número de palabras contado, de forma similar al procedimiento burocrático empleado en las universidades anglosajonas con la entrega de "assignments". El número de formatos utilizados es muy extenso, con lo que no suele haber problemas de incompatibilidad (.txt, .doc, .docx, .pdf, etc.).

Y es aquí donde entramos en la médula del sistema; el tratamiento del trabajo para obtener los índices de similitud. El conjunto de algoritmos que utiliza Turnitin, fueron en principio

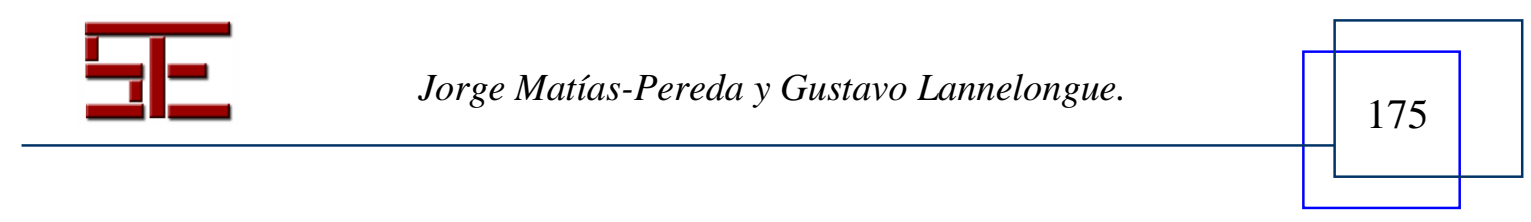




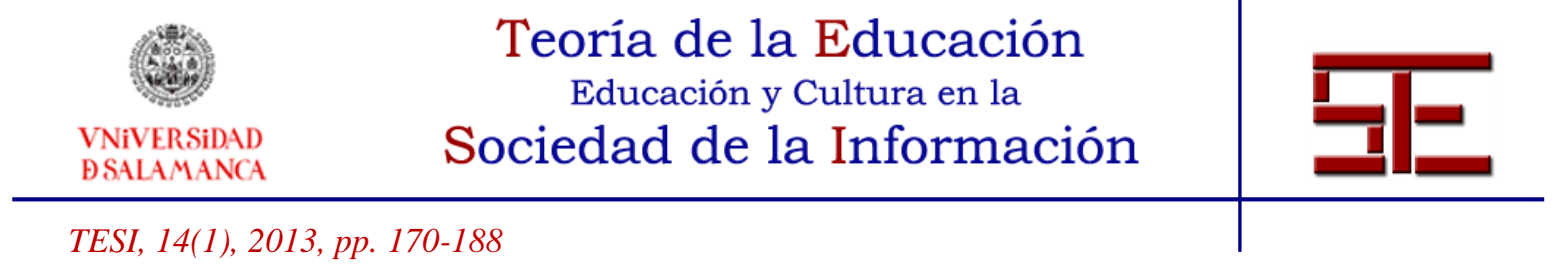

diseñados para analizar las ondas cerebrales (The Economist 2002). En esencia, según su creador, J. M. Barrie (2008), el sistema transforma las palabras del documento en una secuencia de números. El objeto de esta transformación es formar una "huella digital". La huella digital, esas secuencias de números, se ordenan en función de la posición relativa de los caracteres en cada página. El algoritmo analiza cada carácter y su posición en relación con el resto de caracteres y los contrasta con la huella digital de todos los documentos indexados en la base de datos de Turnitin. Cuando el sistema encuentra 40 caracteres idénticos (unas 8 palabras) que coinciden, entonces determina la posición de la huella emparentada y la ubica mostrando la fuente en el informe de similitud.

Una vez que el informe de veracidad se ha generado, se obtiene el primer reporte inicial. En él podemos ver el nombre del trabajo correspondiente de cada alumno, el número de identidad, la fecha de envío y el porcentaje de similitud encontrado. Los trabajos que presentan un más alto grado de similitud se marcan en colores naranja y rojo, los que tienen un menor grado en azul y verde, siendo el punto intermedio reflejado en color amarillo $^{1}$. En particular, podemos enfrentarnos a las siguientes situaciones:

- Articular un envío doble, uno a través de la plataforma en la que se sustente el aula virtual de la asignatura y el otro a través de Turnitin. En este caso tenemos un duplicado del trabajo. Algunos alumnos pueden enviar un trabajo no plagiado, pero carente de contenido útil a la plataforma Turnitin y un trabajo plagiado al aula virtual, sabiendo que la calificación la reciben a través del aula virtual.

- Articular un envío único a través de Turnitin (esta ha sido nuestra opción), con ello evitamos el supuesto anterior, pero es necesario corregir el trabajo sobre el informe de veracidad o sobre la copia del trabajo subida a Turnitin, con lo que, por ejemplo, no es posible utilizar el procesador de textos para reenviar el trabajo con notas de comentario a no ser que se cuente con el módulo Grademark, ofertado conjuntamente con Turnitin2 por el fabricante.

- Articular un envío único a Turnitin a través de la plataforma donde se aloje el aula virtual. Tiene el mismo inconveniente que la segunda opción, es decir, no podemos devolver el trabajo por correo electrónico con comentarios, pero nos aseguramos de que existe un trabajo único y que este es accesible desde la plataforma de uso común de la asignatura.

Una buena opción es permitir que el alumno suba un borrador de su trabajo y que tenga acceso al informe de similitud previamente a entregar la copia definitiva. Esta opción es adecuada en trabajos tutorizados. No obstante, en nuestro caso no elegimos esta opción,

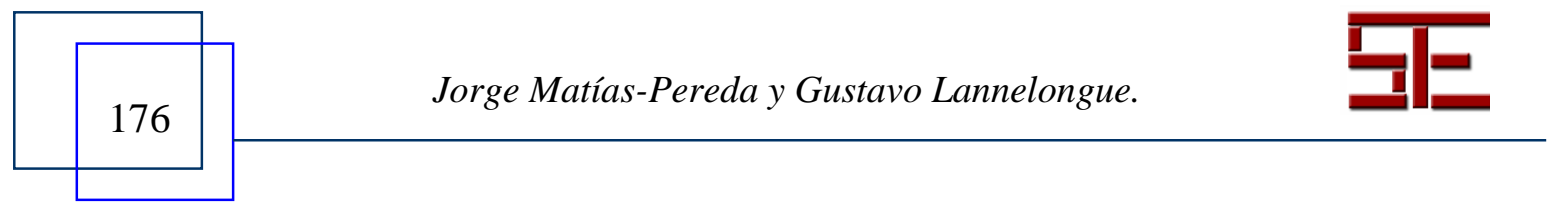




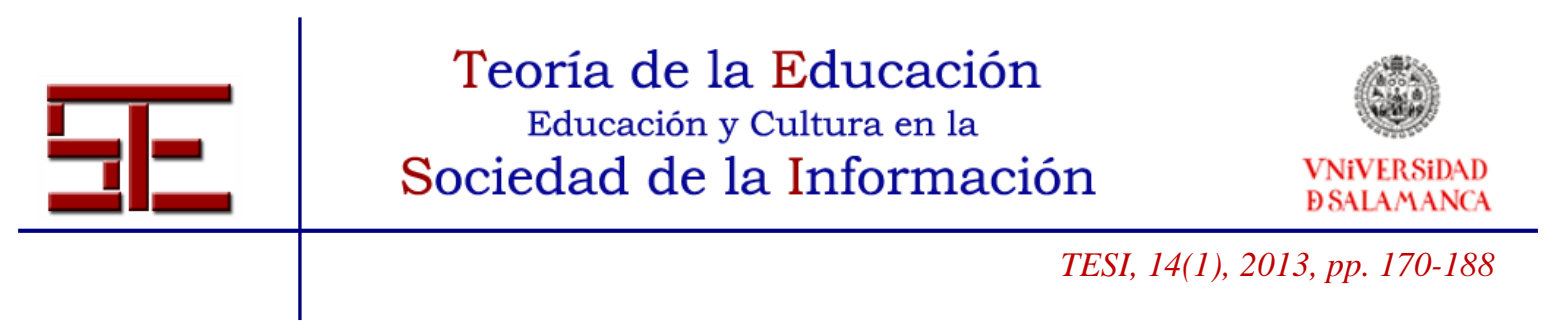

debido a que la mayoría de los alumnos entregaban sus trabajos en las últimas horas del último día permitido, con lo que la parte más importante de la comprobación, la verificación contra otros trabajos, no podía producirse.

Cuando abrimos un informe definitivo de similitud, lo que nos encontramos es una doble página. En la parte izquierda aparece el trabajo enviado por el alumno (su copia digital) y en la derecha, las fuentes numeradas por orden de mayor a menor similitud encontrada y clasificadas por colores. De esta forma es sencillo comprobar qué parte del trabajo del alumno corresponde con qué fuente ${ }^{2}$. Podemos abrir la fuente (si es una página de Internet de acceso público) o bien solicitar la visualización del documento a la institución o autor del cual procede (por ejemplo un trabajo de otra universidad).

Es en este momento donde entra en juego el criterio del profesor. Cada trabajo o práctica, en función de la naturaleza de la misma, requerirá un criterio de corrección distinto. Podemos considerar a Turnitin una buena herramienta que nos permite tener mayor información sobre la veracidad del trabajo entregado, pero tenemos que ser conscientes de que sólo una revisión en profundidad puede determinar el mayor o menor grado de similitud. Las cifras son siempre orientativas en lo que al porcentaje de similitud se refiere. Así, es posible que un trabajo donde obligatoriamente se inserten frases estándar relativas a algún asunto de la práctica nos dé un falso porcentaje de similitud y todos estos aspectos deben ser considerados de forma individual en cada corrección.

Una vez en el proceso de corrección, podemos hacer dos ajustes adicionales. Si el trabajo incorpora citas de otros autores, es posible eliminar aquella parte del texto referenciada del informe de veracidad, con lo que volvemos a obtener unas cifras distintas en los porcentajes de similitud. Si el trabajo tiene una parte final con la bibliografía empleada, debemos activar la eliminación del control de veracidad sobre esta parte. Lo mismo que en el caso de las citas, existe una opción que debemos marcar para eliminar la bibliografía en el reporte de veracidad definitivo, ya sea de forma individual o en la página preliminar para todos los trabajos de la carpeta.

\section{4.- TURNITIN FRENTE A LOS MEDIOS TRADICIONALES ANTICOPIA}

El docente cuenta con pocos medios para verificar la autenticidad de los trabajos entregados por los alumnos. Circulan en Internet una serie de listas de chequeo de acciones que debería comprobar el docente para evitar el plagio que necesariamente requieren un consumo de tiempo muy importante por parte del profesor. Estas listas de

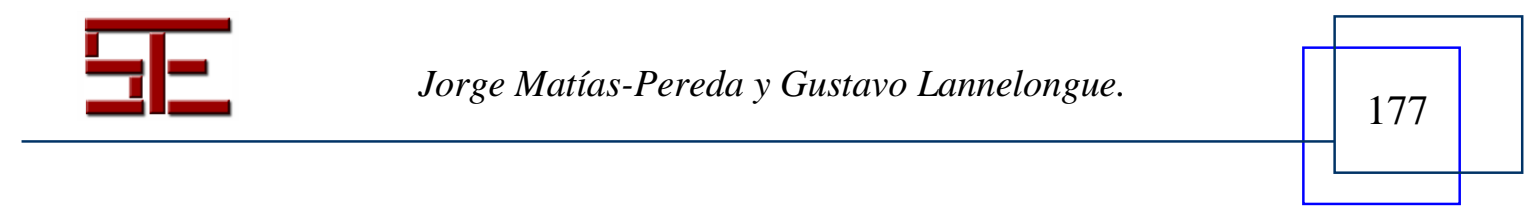




Teoria de la Educación
Educación y Cultura en la
$\begin{gathered}\text { VNiVERSIDAD } \\ \text { BSALAMANCA }\end{gathered}$

chequeo pasan por el examen minucioso de elementos sospechosos de no haber sido redactados por el alumno, tales como discordancia en el estilo de redacción entre párrafos o apartados, tipos de letra distintos (sinónimo de la existencia de un corta y pega), citas que no se corresponden con documentos disponibles en biblioteca, etc.

Cuando alguno de estos elementos es detectado por el profesor, la herramienta más comúnmente utilizada para comprobar efectivamente que se trata de un plagio es la inclusión de parte del texto sospechoso en un motor de búsqueda de uso general. Todo ello lleva una gran cantidad de tiempo, que puede ser reducido, al tiempo que se reduce la probabilidad de copia y se mejora el análisis con un sistema anticopia del tipo Turnitin.

\section{5.- RESULTADOS DE APLICACIÓN EN EL AULA}

En esta sección queremos mostrar cuáles han sido los principales resultados obtenidos con el uso de Turnitin en algunas de nuestras clases. Para ello presentamos datos recogidos en los cursos 2006-2007 y el 2008-2009 en las asignaturas de Información, Organización y Control ( $4^{\circ}$ de ADE) y de Dirección de la Producción y de la Información ( $3^{\circ}$ de ADE) de la Universidad de Salamanca. La primera de ellas sólo está formada por un grupo, la segunda por tres grupos (ver Tabla 1). Los alumnos conocían con anterioridad que se iba utilizar este programa para verificar sus trabajos. Se dedicó en todas las asignaturas al menos 45 minutos de clase presencial a la explicación del funcionamiento de Turnitin, de las técnicas no admitidas (como por ejemplo copiar y pegar párrafos o trabajos escritos en otros idiomas y luego traducidos, la utilización de sinónimos para evitar la detección, etc.). En la misma clase presencial se recalcó la importancia de la ordenación y síntesis de los argumentos, la expresión personal de las ideas y el estándar de citación admitido en cada prueba. De todo ello, aparte de la propia charla en el aula, se dejó abundante material de consulta en el aula virtual de las asignaturas.

\begin{tabular}{|ll|}
\hline \multicolumn{2}{|l|}{ Tabla 1: Distribución por grupos } \\
\hline & $\mathrm{N}^{\mathbf{o}}$ de trabajos \\
\hline Grupo 1 & 54 \\
\hline Grupo 2 & 18 \\
\hline Grupo 3 & 19 \\
\hline Grupo 4 & 88 \\
\hline Total & 179 \\
\hline
\end{tabular}

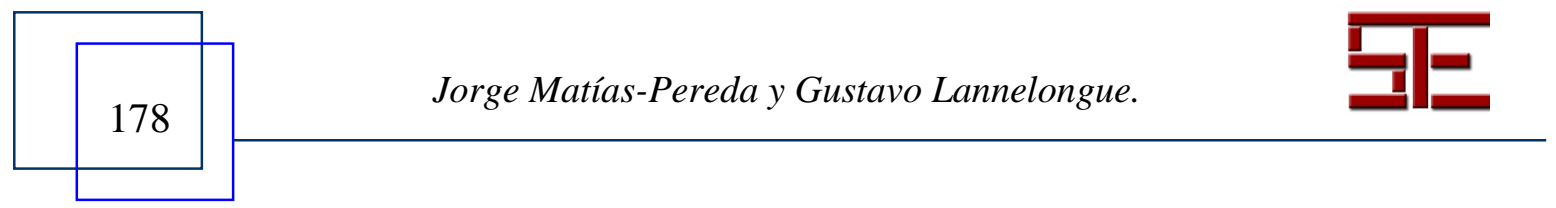




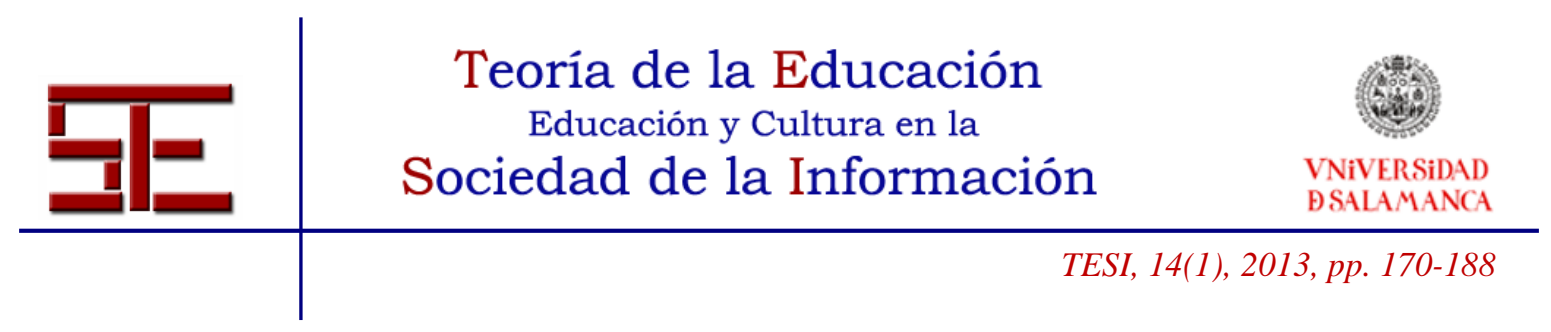

Los datos que presentamos reflejan las puntuaciones obtenidas en el índice de similitud de Turnitin por los cuatro grupos en dos trabajos entregados en momentos distintos por los alumnos. Todos los trabajos eran de similares características (eran estudios de caso de empresas y su longitud no debía ir más allá de las 4 páginas). Por lo que, independientemente de la asignatura, hablaremos de 4 grupos. Todos los alumnos hicieron las dos entregas. Los resultados obtenidos muestran como la mayoría de las puntuaciones se encuentran entre 0 y 20. Hay que resaltar que obtener 0 en el índice de similitud es complicado por la propia dinámica del algoritmo, por lo que aunque consideramos que es importante revisar todos los trabajos, estas puntuaciones pueden considerarse normales, lo que significa que en esos trabajos no deberían encontrarse indicios de plagio. La media para el primer trabajo fue de 7,54 y para el segundo de 5,83 (ver Tabla 2).

Tabla 2: Estadísticos descriptivos

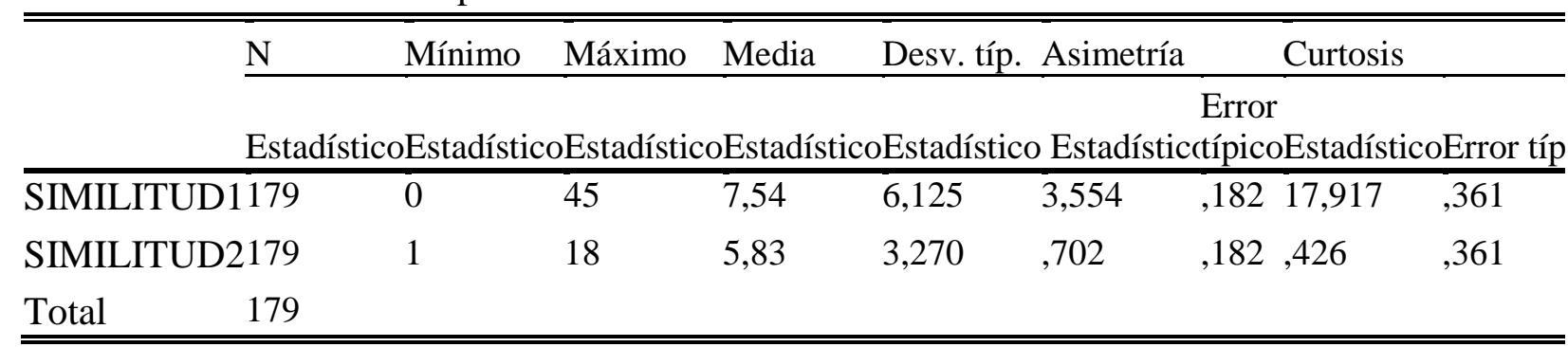

Por lo tanto nuestra atención se centró en los casos con más de 20 puntos en el índice de similitud que fueron en el primer trabajo seis y en el segundo cero (ver Figura 1). Todos los casos en esa situación, excepto uno, presentaban un porcentaje inferior al 3\% de similitud en cada una de las fuentes señaladas en el informe de veracidad. Esto quiere decir que Turnitin no pudo apreciar más de un 3\% de similitud entre documentos en su base de datos y el trabajo entregado. El alto valor se debía, por tanto, a muchas fuentes de similitud, pero con un baja significatividad en cada una de ellas a la hora de explicar los posibles indicios de plagio. El único trabajo que sí fue penalizado presentaba un escaso número de fuentes, pero un alto porcentaje de similitud en cada una de ellas, hasta llegar a ese $45 \%$. La penalización aplicada en este caso, y recogida en las normas de evaluación de la asignatura, era la obligatoriedad de realizar el examen en convocatoria oficial, no pudiendo seguir a partir de ese momento el alumno la evaluación continua.

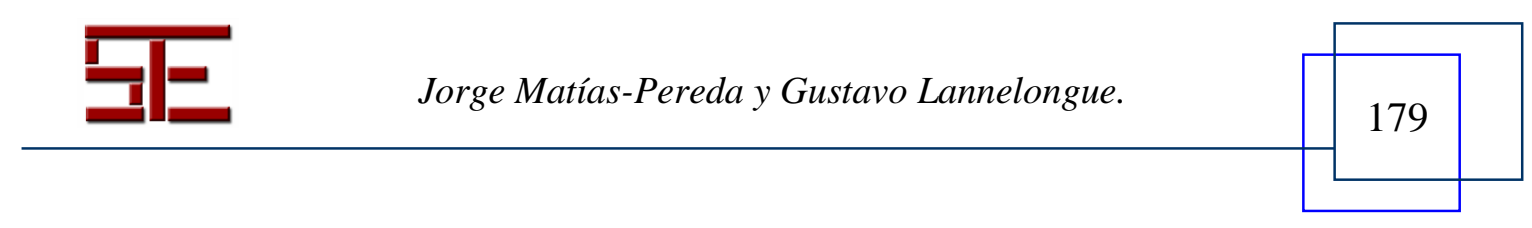



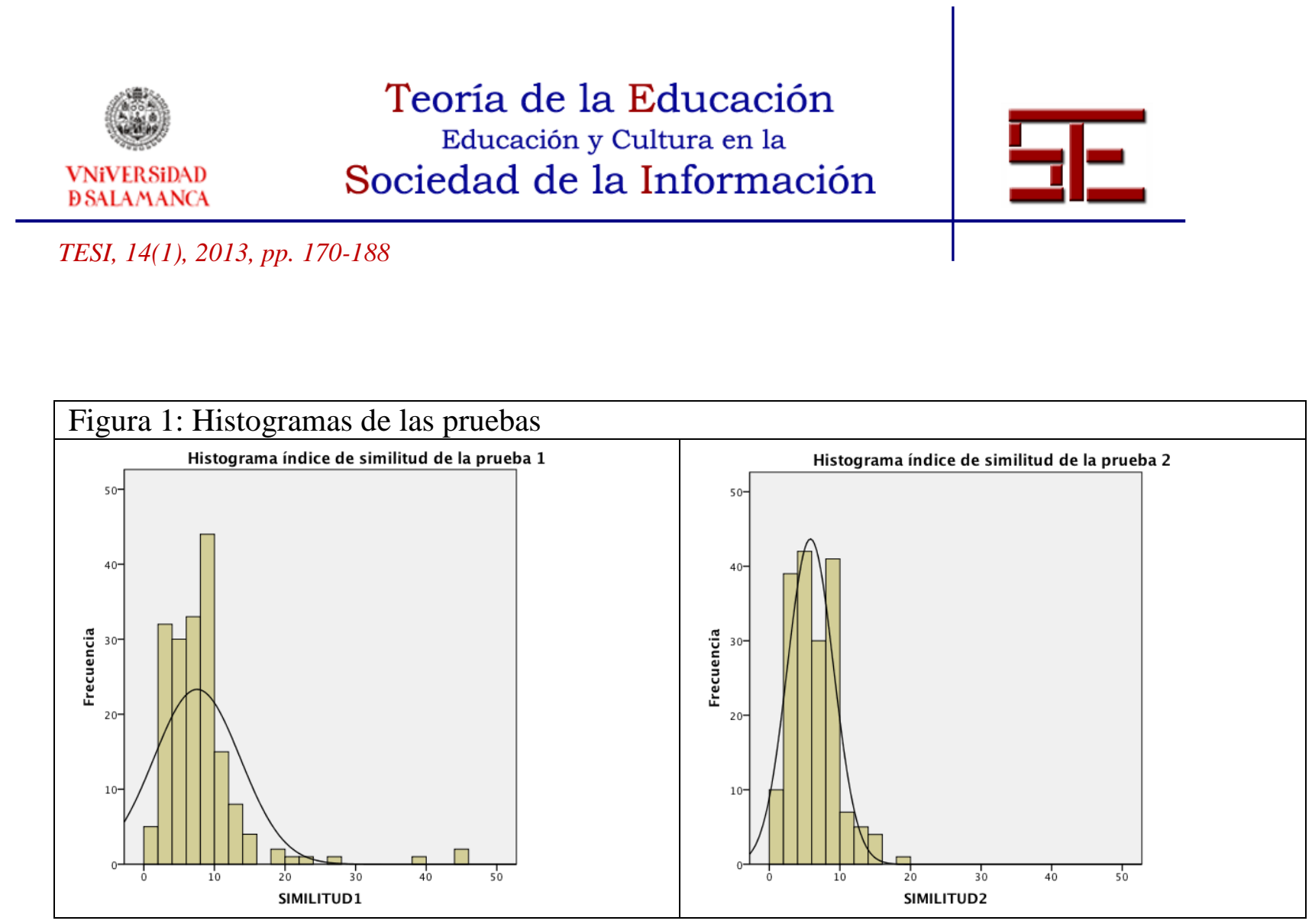

Como se puede apreciar en los histogramas (ver Figura 1) la distribución de resultados es más homogénea en la segunda entrega, aproximándose sus valores de asimetría y curtosis al de una distribución normal (ver Tabla 1). La media de esta segunda entrega es también menor $(5,83$ por 7,54$)$, lo cual podría sugerir un efecto aprendizaje por parte de los alumnos. Esto podría deberse a que preparasen mejor sus trabajos según las reglas (aprender a escribir según las reglas) o que escribiesen pensando más en la herramienta (escribir trabajos ad hoc). Este efecto puede verse en la Figura 2, que muestra la puntuación agregada de similitud de todos los casos.

Figura 2: Puntuaciones acumuladas en las pruebas
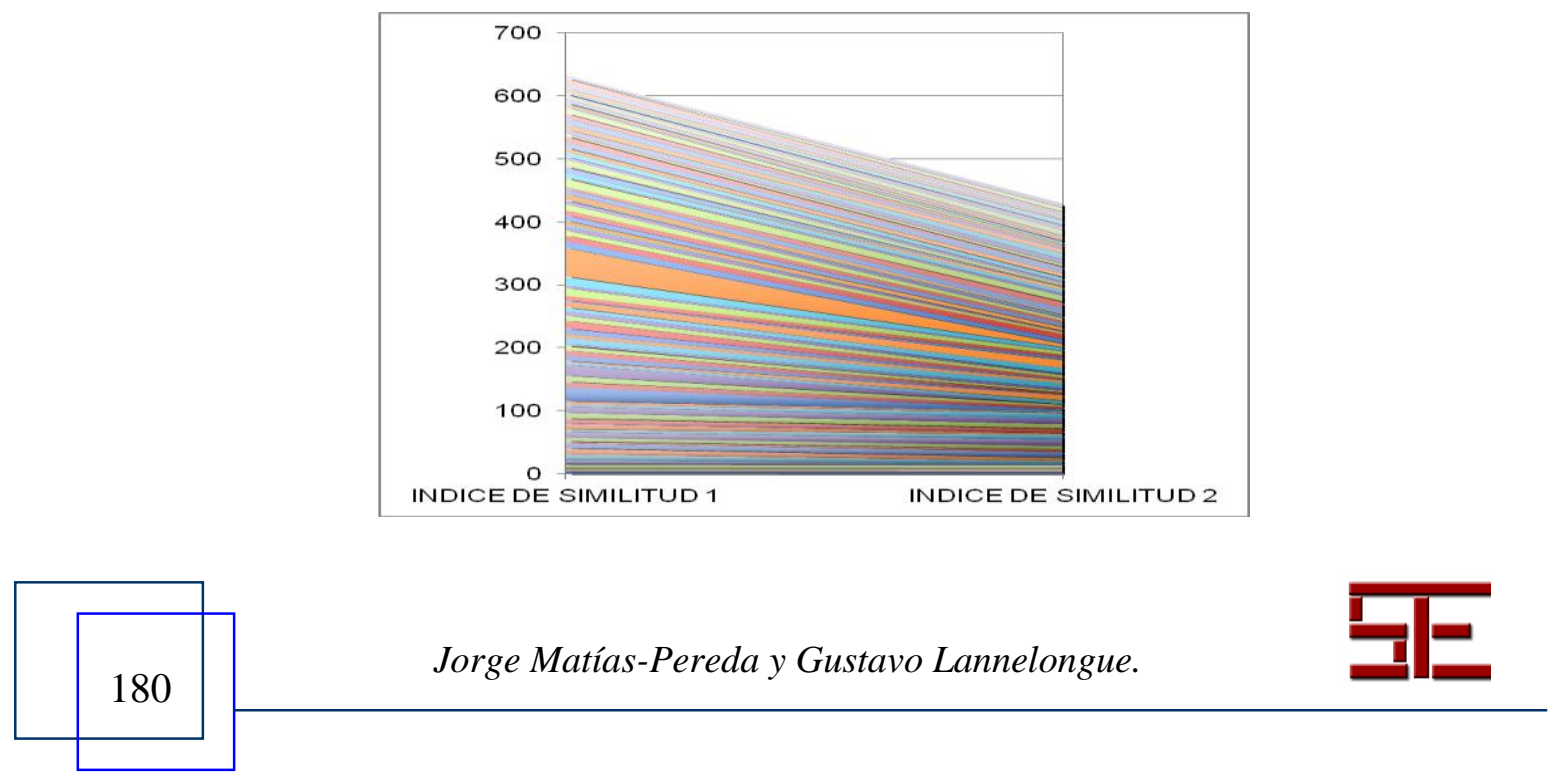


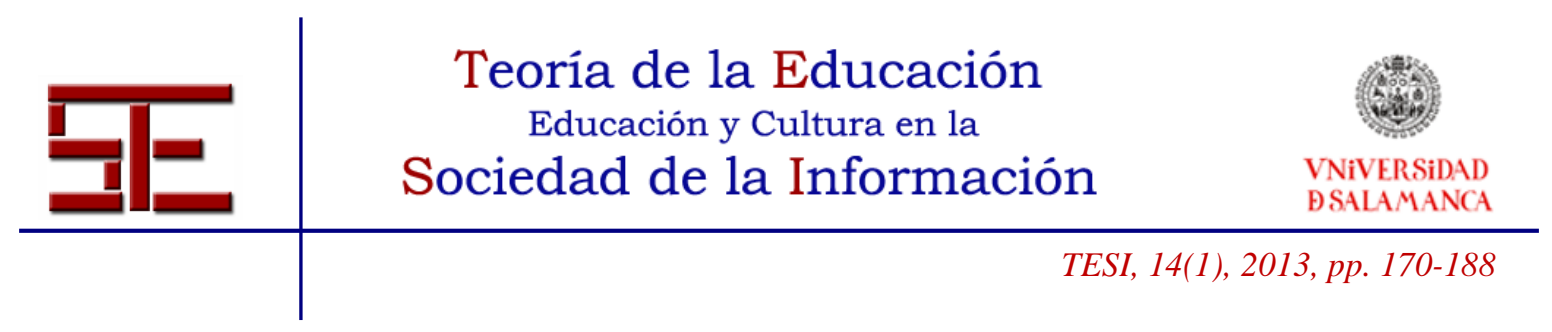

En segundo lugar, para complementar los resultados de la comparación de entregas, se lanzó una encuesta a alumnos de la asignatura: Información, Organización y Control que ya habían tenido una experiencia previa con Turnitin, obteniendo un número de 31 contestaciones útiles al cuestionario. Como mínimo, los alumnos encuestados entregaron 3 trabajos y un máximo de 9 a través de Turnitin, con una media de 6,3 trabajos entregados por alumno. La encuesta recogió 22 ítems divididos en cuatro categorías que pretendían recoger las causas y el nivel de plagio antes y después de Turnitin por una parte y, por otra, los problemas y mejoras percibidos del uso del sistema por parte de los alumnos. Los ítems se escogieron a través de una revisión de la literatura (Hauptman, 2002; Weinstein y Dobking, 2002; Beasley, 2004; Wheeler, 2007) que analiza las causas de plagio en alumnos universitarios.

Como cifra destacada, se encuentra el nivel de plagio declarado por los alumnos antes y después de Turnitin. Antes de Turnitin el plagio reconocido por los alumnos asciende a un $38,5 \%$, mientras que con el uso de Turnitin, éste se reduce hasta un 4,1\%.

Entre las principales causas manifestadas por los alumnos que les indujeron a plagiar antes de la implantación de Turnitin fueron, por orden de mayor a menor, las siguientes:

- (30\%) Falta de tiempo para entregar los trabajos.

- (20\%) Conocimiento de que el profesor aplicaba criterios de corrección superficiales:

- $(19,4 \%)$ Complejidad de los trabajos muy elevada.

Mientras que las principales causas de plagio al tiempo que se utilizaba la licencia Turnitin eran otras completamente diferentes:

- $(3,2 \%)$ Encontrar formas de burlar al sistema.

- $(3,2 \%)$ Encargo de trabajos a otras personas.

Se observa por tanto una modificación radical en el comportamiento ante el plagio manifestada por los propios alumnos antes y después de la utilización de Turnitin.

En cuanto a la mejora percibida con el uso de Turnitin, los alumnos argumentaron como mejoras principales percibidas, las siguientes:

- (51,6\%) Incrementa la capacidad de colaboración en uso conjunto con foros electrónicos.

- $(48,4 \%)$ Redacción de textos más elaborados, claros y sintéticos.

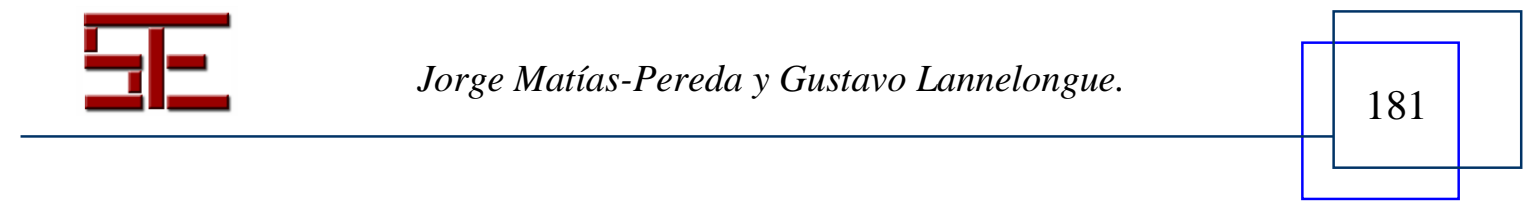




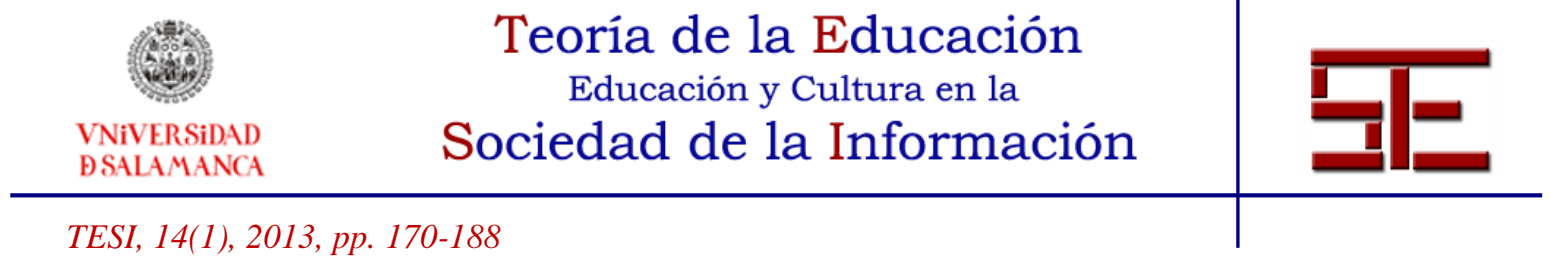

- (45,2\%) Mejora de la capacidad de búsqueda de información.

El problema principal percibido fue la dificultad que los alumnos tenían para citar correctamente, ya que el $48,4 \%$ de los alumnos manifestaron graves dificultades para adquirir habilidades de citación correcta.

Por otro lado, los alumnos manifestaron en un $67,7 \%$ haber utilizado aplicaciones gratuitas disponibles en Internet para comprobar la veracidad de sus trabajos. Lo que pone de manifiesto la preocupación que tienen los alumnos con el uso del sistema.

\section{6.- VENTAJAS E INCONVENIENTES DEL USO DE TURNITIN}

A continuación enumeraremos una serie de ventajas e inconvenientes que se pueden producir con el uso de Turnitin como herramienta de control del plagio, a la luz de la experiencia acumulada por su uso.

\section{1.- Ventajas del uso de Turnitin}

Mejora de la expresión escrita

Uno de los requisitos importantes en el uso de Turnitin es la necesidad que el alumno, para evitar la penalización por plagio, tiene de escribir con sus propias palabras, extraídas de un proceso de reflexión y síntesis previo, el trabajo que va a entregar para ser calificado.

Por lo tanto uno de los efectos más inmediatos es la mejora en la calidad de la redacción y expresión escrita de los trabajos entregados. Efectivamente, el alumno hace consciente la necesidad de cuidar su expresión escrita a través del poder disuasorio de la penalización. Turnitin se convierte en ese elemento disuasorio. De la fuerza con que la disuasión y la concienciación del problema se fijen en la mente del alumno depende su impacto más o menos productivo sobre las capacidades de éste. Por lo tanto es fundamental que el docente instruya primero y recalque después la necesidad de una reflexión sobre el modo en que el alumno redacta sus escritos, al mismo tiempo que supervisa a aquellos alumnos con problemas en esta área.

Mejora de la creatividad

No sólo es la forma en que expresemos nuestras ideas sobre el papel el elemento crítico

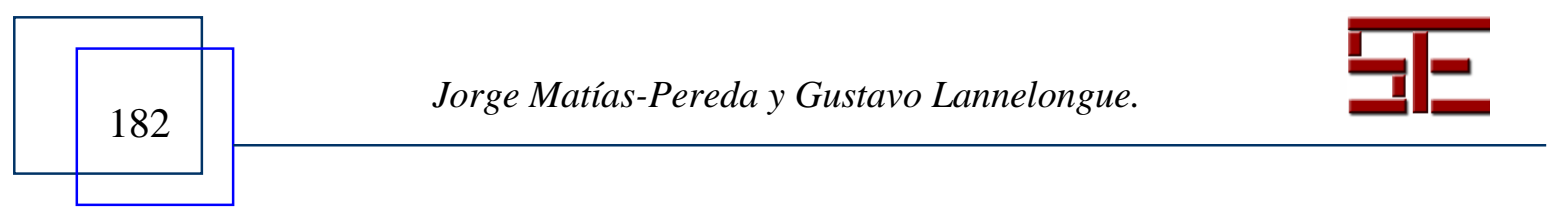




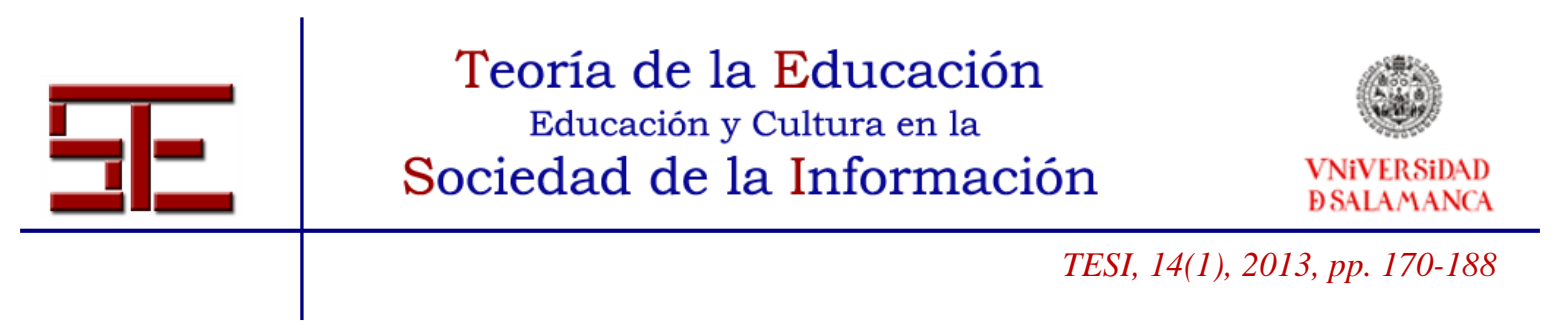

para evitar la penalización, sino que, al hacerlo, estamos obligando al establecimiento de un proceso de análisis y reflexión sobre los contenidos de los trabajos y prácticas a entregar. Pasamos de un papel pasivo, puramente recopilatorio de información, a un papel activo, donde se requiere la participación del alumno. En este sentido es útil proporcionar tareas donde el alumno tenga que analizar y/o proporcionar una crítica a conceptos establecidos. Una tarea del tipo "construir una lección sobre un tópico o concepto" puede llevar al alumno a un plagio inintencionado y a limitar su capacidad de expresión escrita y su creatividad (Abasi, et. al. 2006).

Refuerzo de la autoridad del profesor

El establecimiento de unos parámetros, en principio poco discutibles, sobre el comportamiento ético del alumnado al incorporar una herramienta como Turnitin, es aceptado sin problemas (en nuestra experiencia) por todos los alumnos. La existencia de normas claramente explicitadas desde el comienzo del curso académico es siempre positiva, ya que reduce la incertidumbre sobre la actuación del alumno y proporciona un marco razonable para la solución de conflictos que se puedan producir. De esta forma, si el alumno es penalizado por plagio, siempre es posible remitirnos a las reglas de actuación para ese caso cuando citemos al alumno en cuestión. El informe de veracidad es el documento que corrobora la penalización, que debidamente explicada una vez oídas las alegaciones del alumno, da lugar a la sanción. En este sentido es importante recalcar que una heterogeneidad en las penalizaciones aplicadas ante mismos niveles de plagio puede introducir un elemento distorsionador en el marco ético de referencia del alumno (Kenny, D. 2008). Es deseable la existencia de un criterio único, dado a conocer y plenamente disponible para el alumno de aplicación por todos los profesores que utilicen el sistema.

Refuerza el valor del esfuerzo del alumno

La puntuación de trabajos y prácticas debe premiar el esfuerzo del alumno. Todos los alumnos conocen la facilidad con que es posible recopilar información en Internet para confeccionar un trabajo, por lo tanto el conocimiento de que no son posibles comportamientos oportunistas (que pueden dar como resultado una injusticia en la valoración del esfuerzo) da un valor adicional al esfuerzo y extiende el sentimiento de una aplicación homogénea de los criterios de puntuación que induce un clima más positivo en el desarrollo del proceso de aprendizaje.

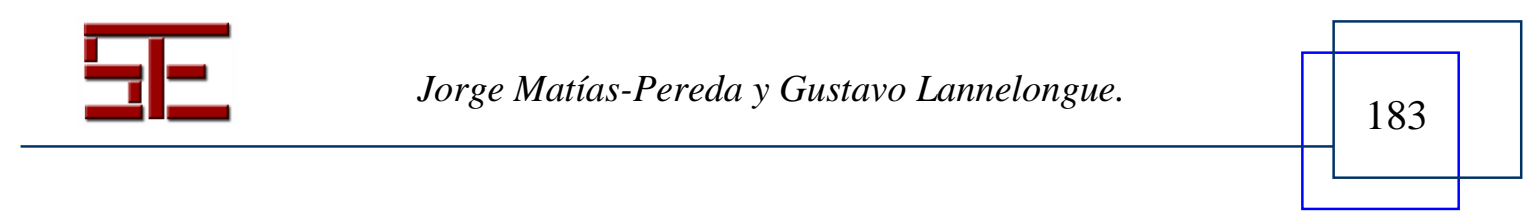




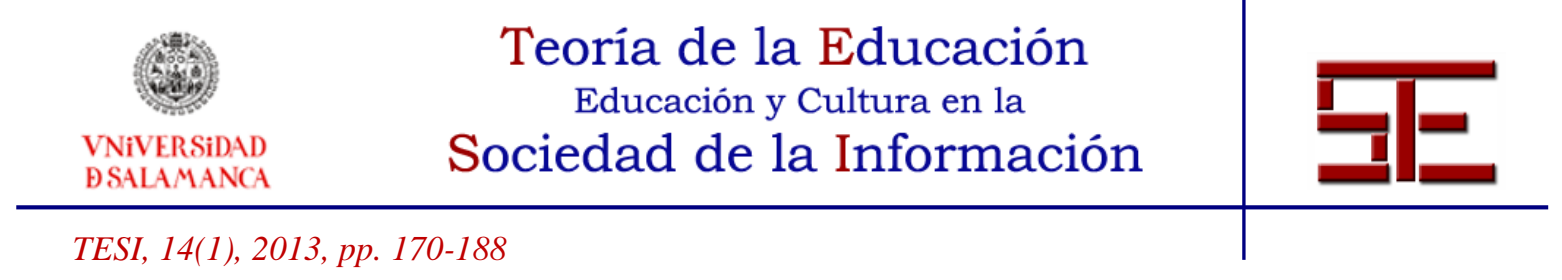

\section{2.- Inconvenientes del uso de Turnitin}

Percepción de Turnitin como elemento represivo

Uno de los problemas principales que puede aparecer con el uso de Turnitin es la percepción de una represión excesiva por parte del alumnado. Creemos, una vez experimentado el uso de Turnitin durante varios años, que esta presión no debe aparecer si el docente es capaz de instruir correctamente al alumno indicándole cuáles son las ventajas y los beneficios que se pueden obtener de la herramienta. Como bien apunta Saunders (1993), la adquisición de la práctica del plagio durante la vida universitaria ofrece muchas oportunidades para que el comportamiento se replique en la vida profesional, por lo tanto, es preciso instaurar una cultura ética, más allá del propio uso de Turnitin.

Un buen número de universidades, especialmente en el Reino Unido y los Estados Unidos, llevan a cabo programas de integridad académica que sientan las bases de conducta del alumnado para ajustarlas a un comportamiento ético. Dichas bases son, la mayoría de las veces, incorporadas a los estatutos de la Universidad. Estos programas son difundidos entre toda la comunidad universitaria. Se organizan seminarios, campañas de sensibilización y talleres cuyo fin es contribuir a los objetivos de integridad de la institución. Qué duda cabe de que estos programas inducen una percepción en la comunidad universitaria que incide directamente en la generación de una cultura donde el comportamiento ético (incluida la evitación del plagio) se encuentra permanentemente presente (Moore-Howard, 2007).

Conflicto con los derechos de autor del alumno

Uno de los problemas principales con que se encuentra el uso de los sistemas antiplagio es la protección de los derechos de autor de los alumnos (Foster, 2002). Turnitin alega que lo que contrasta no son documentos, sino "huellas digitales" de los documentos con otras huellas digitales que se encuentran almacenadas en sus bases de datos (www.turnitin.com). Sin duda es un tema complejo, sobre el que existe un encendido debate. En nuestra experiencia, podemos precisar que no hemos tenido ninguna queja en este sentido por parte de los alumnos.

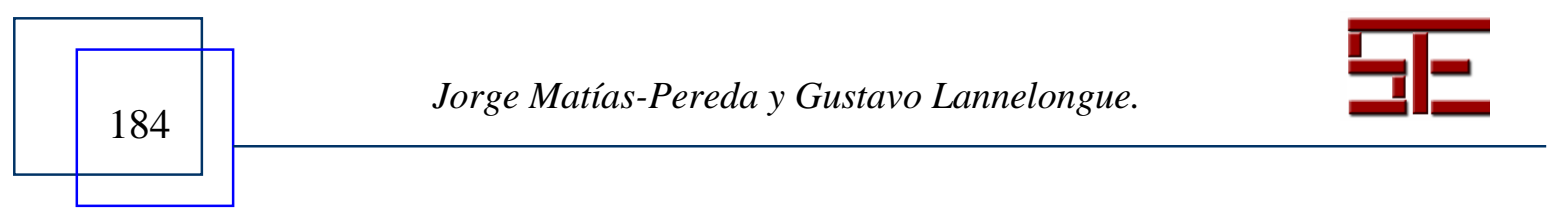




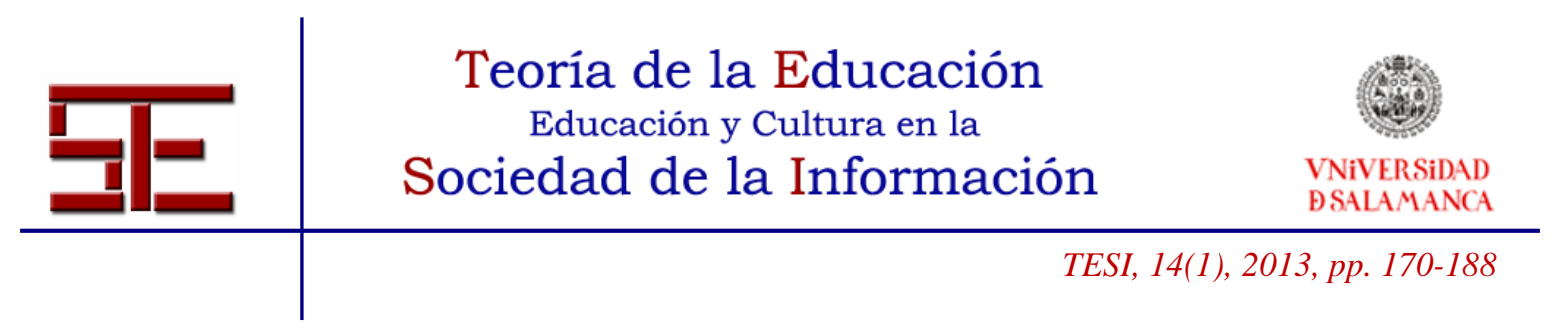

Utilización de Casos de Estudio y prácticas de uso extendido

Es una práctica habitual en las asignaturas de Organización de Empresas y Dirección Estratégica la utilización de casos incluidos en libros de texto y/o la compra a otras instituciones (p. e. Hardvard Publishing) de los casos que se utilizan en el aula. Tenemos que estar advertidos de que, si hacemos esto, es posible que los índices de similitud se disparen. ¿Cuál es la razón? Es simple. No es otra que el envío durante una gran cantidad de años de trabajos de cientos de estudiantes sobre el mismo caso. Es difícil que las preguntas o el enfoque sea idéntico para un profesor determinado o para una universidad. Afortunadamente para las universidades de habla castellana, éste es, todavía, un problema secundario, ya que la mayoría de los ensayos y trabajos sobre esos casos suelen proceder de universidades anglosajonas. Pero, de extenderse el uso de Turnitin y otras herramientas similares, como parece ser el caso, este problema podría ser importante de aquí a no muchos años. De ahí que sea la propia habilidad del docente de plantear nuevos enfoques y preguntas sobre los casos una cuestión necesaria para evitar este problema.

Baja motivación del profesorado a la adopción de la licencia

En nuestra experiencia, el número de profesores que utilizaron de forma completa la licencia fue muy reducido, ascendiendo tan solo a 3 profesores, mientras que la asistencia a los seminarios fue de 16 profesores y unos 10 utilizaron la herramienta de forma experimental sin llegar a utilizarla en el aula de forma práctica. Una vez adquirida la licencia, se impartieron dos seminarios sobre el funcionamiento de Turnitin y se ofreció asistencia a través de correo electrónico y de forma presencial para consultas. La baja implicación del profesorado en su utilización podría deberse, tal y como afirma Younghwa (2010), a una falta de percepción de la efectividad del sistema y también a la creencia de que su manejo pueda ser lo suficientemente complicado para explotarlo correctamente, o bien a que el plagio no se percibe como una seria amenaza a la integridad académica. En este sentido sería útil poder ofrecer una información completa a todos los docentes sobre las ventajas e inconvenientes del uso de la licencia y no solo a aquellos que muestran una predisposición a su utilización.

\section{7.- CONCLUSIONES}

Las principales conclusiones que extraemos del uso del sistema anticopia Turnitin son positivas y hemos comprobado cómo los alumnos han tomado consciencia de la existencia de esta herramienta en el proceso de evaluación, lo cual ha provocado por su

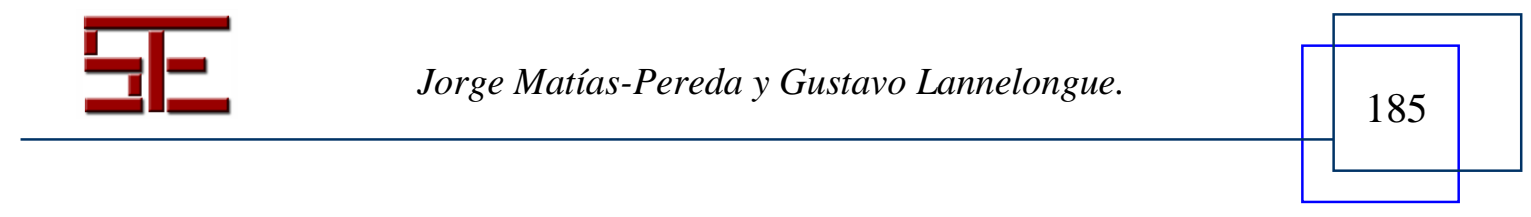




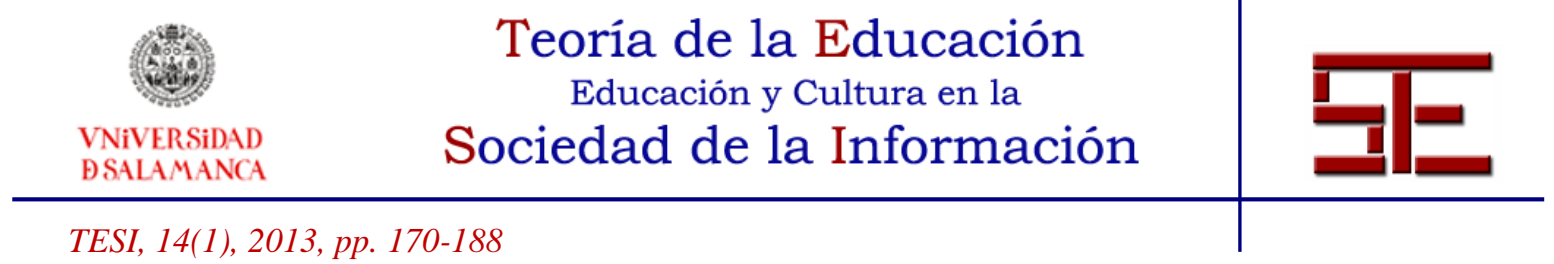

parte una mayor atención y dedicación a la expresión escrita y al desarrollo de ideas y conceptos propios. También ha permitido que disminuyan las sospechas sobre la autenticidad de los trabajos, valorizando el esfuerzo real de los alumnos, mejorando su relación con el profesor y reforzando la autoridad de este último. La incorporación de este sistema también puede conllevar algún aspecto negativo como la generación de un sentimiento de rechazo por parte del alumno por la percepción de un control exagerado, la adaptación del trabajo escrito a los requerimientos de Turnitin (limitando la creatividad y el enfoque crítico) y problemas derivados de utilizar software de empresas privadas por su precio y por el vacío legal que rodea la difusión de trabajos de alumnos.

Los sistemas antiplagio no son infalibles y sus fisuras van extendiéndose a medida que los alumnos desarrollan habilidades y estrategias para contrarrestar la aparente seguridad del sistema. Sin duda son una herramienta de extrema utilidad para detectar los casos más típicos de plagio, pero existen lagunas en la reproducción de conceptos que son utilizados como propios, modificando la redacción de los enunciados originales, imperfección en el control del uso de sinónimos, etc. No podemos, de esta forma, confiar en dicha herramienta como un elemento único y aceptable para orientar el trabajo ético y sistemático del alumno, pero sí constituye una herramienta de indudable valor para el docente en su función de supervisor ético y garante del esfuerzo real del alumno.

\section{8.- BIBLIOGRAFÍA}

Anon. (2002). Plagiarise: let no one else's work evade your eyes. [Versión electrónica]. The Economist, 14 marzo. Extraído el 10 de enero, 2012, de http://www.economist.com/node/1033832.

Abasi, A. R, Akbari, N. y Graves, B. (2006). Discourse appropriation, construction of identities, and the complex issue of plagiarism: ESL students writing in graduate school. Journal of Second Language Writing, 15, 102-117.

Beasley, J. D. (2004, marzo). The impact of technology on plagiarism prevention and detection: research process automation, a new approach for prevention. Ponencia presentada en Proceedings of the Plagiarism: Prevention, Practice and Policies.

Foster, A. L. (2002). Plagiarism-detection tool creates legal quandary: when professors send students' papers to a database, are copyrights violated? Chronicle of Higher Education, 48, 37-42.

Hauptman, R. (2002). Dishonesty in the academy. Academe, 88, 24-48. Extraído el 15

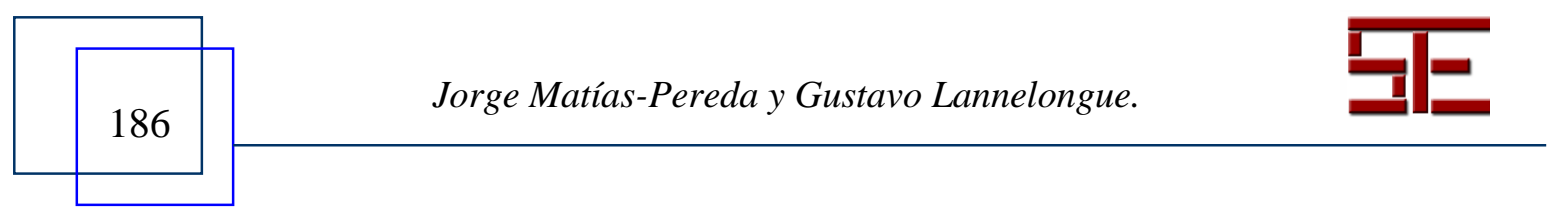




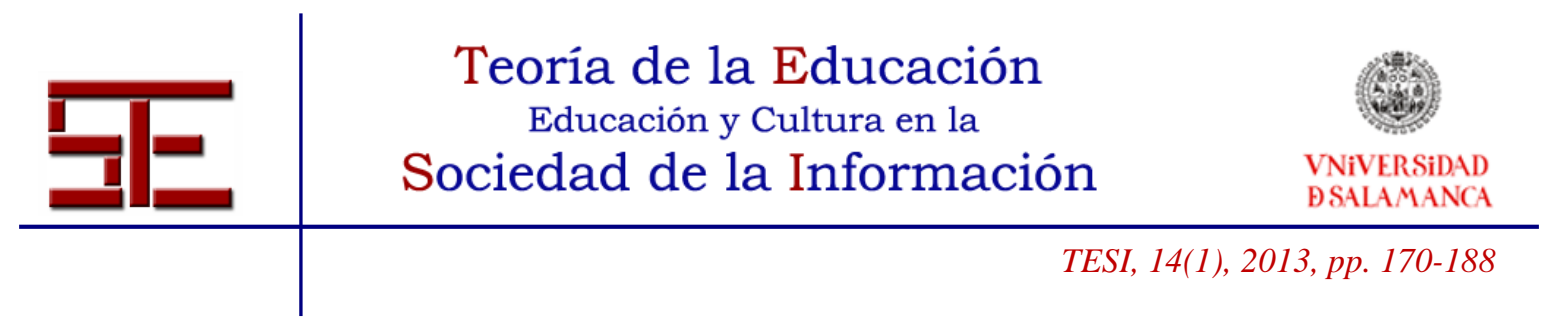

de enero, 2012, de

http://www.aaup.org/publications/Academe/2002/02nd/02ndhau.html.

Kenny D. (2007). Student plagiarism and professional practice. Nurse Education Today, $27,14-18$.

Moore-Howard, R. (2007). Understanding 'Internet plagiarism'. Computers and Composition, 24, 3-15.

Saunders, E. J. (1993). Confronting academic dishonesty. Journal of Social Work Education, 29, 224-230.

Weinstein, J. y Dobkin, C. (2002, enero 21). Plagiarism in U.S. Higher Education: Estimating Internet Plagiarism Rates and Testing a Means of Deterrence. Extraído el 15 de enero, 2012, de http://webdisk.berkeley.edu/ Weinstein/WeinsteinJobMarketPaper.PDF.

Wheeler, G. (2009). Plagiarism in the Japanese universities: Truly a cultural matter? Journal of Second Language Writing, 18, 17-29.

Younghwa, L. (2011). Understanding anti-plagiarism software adoption: An extended protection motivation theory perspective. Decision Support Systems, 50, 361-369.

\section{9.- NOTAS}

* El presente trabajo ha sido financiado parcialmente por el proyecto de investigación SA083A12-1 financiado por la Consejería de Educación de la Junta de Castilla y León y el proyecto ECO2010-21078 financiado por elMinisterio de Economía y Competitividad.

${ }^{1}$ El color azul significa $0 \%$ de similitud encontrada al enfrentar el trabajo con la base de datos de Turnitin, el color verde corresponde al intervalo de 1 al 24\% de similitud, el color amarillo corresponde al intervalo del 25 al 49\% de similitud, el color naranja corresponde al intervalo del 50 al $74 \%$ y el color rojo corresponde al intervalo del 75 al $100 \%$ de similitud.

${ }^{2}$ En la parte superior del informe definitivo de veracidad se incorpora un resumen con el porcentaje por tipo de fuente. En concreto, Turnitin separa tres tipos de fuente de similitud: fuentes de Internet, publicaciones y trabajos de alumnos.

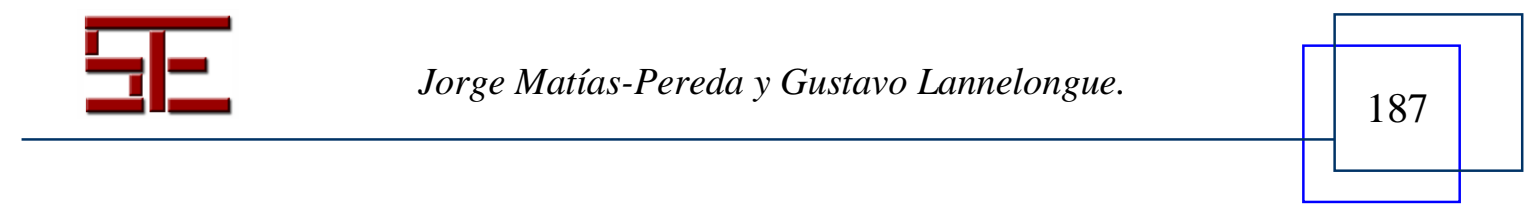




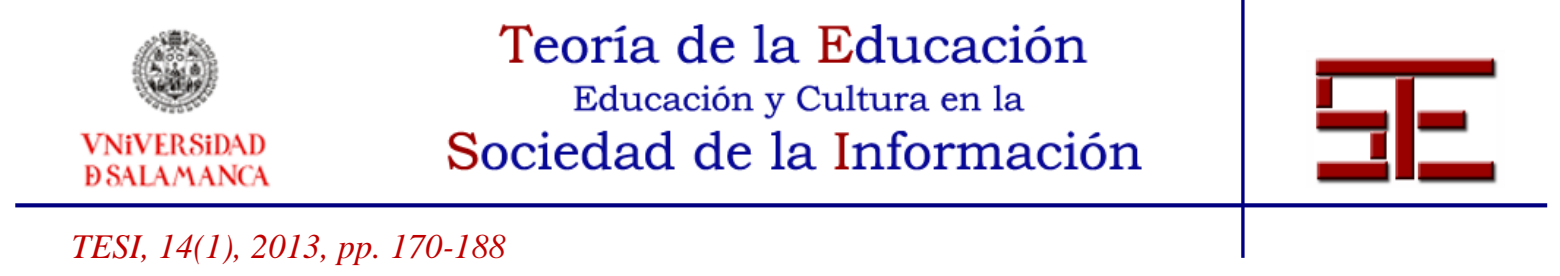

Para citar el presente artículo puede utilizar la siguiente referencia:

Matías-Pereda, J. y Lannelongue Nieto, G. (2013). Técnicas de ayuda en el proceso de aprendizaje: el caso de los sistemas anticopia. Revista Teoría de la Educación: Educación y Cultura en la Sociedad de la Información. 14(1), 170-187 [Fecha de consulta: $\mathrm{dd} / \mathrm{mm} / \mathrm{aaaa}]$.

http://campus.usal.es/ revistas_trabajo/index.php/revistatesi/article/view/9448/9737

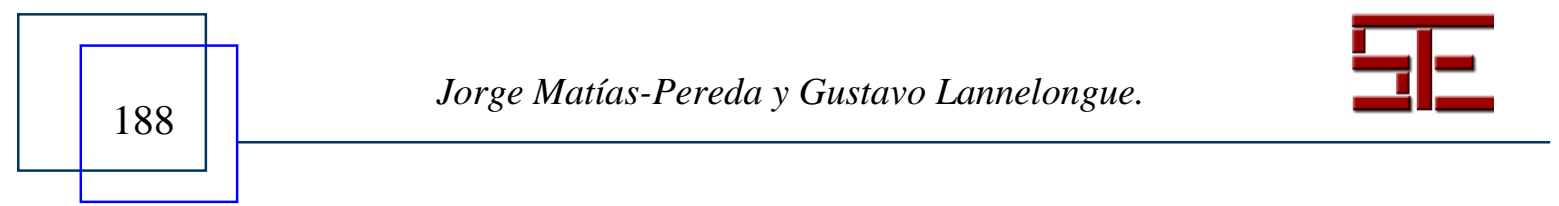

